\title{
Bimanual grasp planning reflects changing rather than fixed constraint dominance
}

\author{
Robrecht P. R. D. van der Wel · David A. Rosenbaum
}

Received: 9 March 2010 / Accepted: 9 July 2010 / Published online: 24 July 2010

(C) The Author(s) 2010. This article is published with open access at Springerlink.com

\begin{abstract}
We studied whether motor-control constraints for grasping objects that are moved to new positions reflect a rigid constraint hierarchy or a flexible constraint hierarchy. In two experiments, we asked participants to move two plungers from the same start locations to different target locations (both high, both low, or one high and one low). We found that participants grasped the plungers symmetrically and at heights that ensured comfortable or easy-tocontrol end postures when the plungers had the same target heights, but these grasp tendencies were reduced when the plungers had different target heights. In addition, when the plungers had different mass distributions, participants behaved in ways that suggested still-different emphases of the relevant grasp constraints. When the plungers had different mass distributions, participants sacrificed bimanual symmetry for end-state comfort. The results suggest that bimanual grasp planning relies on a flexible rather than static hierarchy. Different constraints take on different degrees of importance depending on the nature of the task and on the level of task experience. The results have implications for the understanding of perceptual-motor skill learning. It may be that one mechanism underlying such learning is changing the priorities of task constraints.
\end{abstract}

R. P. R. D. van der Wel ( $\square)$

Donders Institute for Brain, Cognition, and Behaviour,

Radboud University Nijmegen, Nijmegen, The Netherlands

e-mail: r.vanderwel@donders.ru.nl

D. A. Rosenbaum

Department of Psychology,

Pennsylvania State University,

University Park, PA 16802, USA

e-mail: dar12@psu.edu
Keywords Action planning - Bimanual control · Grasping · Object manipulation · Constraint dominance

\section{Introduction}

An essential feature of skilled performance is the ability to adapt to different task demands. Such adaptation involves appreciating what task needs to be performed. For some tasks, it is more important to satisfy some constraints than others. Thus, if one is holding a cup of hot coffee, holding the cup steady is of paramount importance. By contrast, if one has emptied the cup and is about to toss it into a trash can, the precise angle of the top of the cup is obviously of less concern.

These examples suggest that a given constraint can take on different degrees of importance depending on what needs to be achieved. Indeed, what needs to be achieved can be said to reflect the weighting of the constraints to be satisfied and thereby to define the task's internal representation (Rosenbaum et al. 1995, 2001). That said, some constraints may be more heavily weighted than others all or almost all of the time. In the two cases of cup-carrying just mentioned, for example, the person carrying the glass needs to stay upright in both task scenarios.

These observations suggest that an important challenge for a theory of physical action planning is determining which constraints take precedence over others and when they do. We use the term "constraint" to refer to any factor that limits behavior. We use the term "constraint" to refer to factors that may be physically or cognitively limiting. Cognitive constraints are manifested in problem-solving (e.g., in playing chess) and in language use, where rules, either of an explicit or implicit sort, stipulate what word orders are allowed, what phonological 
transitions are permitted, and so on (Prince and Smolensky 1997).

We will say that if one constraint is always more important than another, the relation reflects strict dominance. Another possibility is that the relative importance of constraints changes depending on task circumstances. We will say that if one constraint is not always more important than another, the relation between the constraints reflects changing dominance. The question we pursued in this study was which of these relations applies to a pair of motor-control constraints related to object manipulation. One constraint pertains to end-state comfort. The other pertains to bimanual symmetry. We will discuss both constraints below before turning to the experiments we did to identify the nature of their dominance relation, strict or changing.

The end-state comfort effect was discovered by the second author in a restaurant. While having a meal, he spotted a waiter filling glasses with water. Each glass was inverted to begin with, so each glass had to be turned right-side up before the waiter could pour water into it. What struck the second author was that the waiter picked up each glass with his thumb pointing down. Lifting a glass with a thumbdown posture is a strange thing to do when considered out of context, but the behavior made sense in this context. By lifting each glass with the thumb down, the waiter could later hold the glass in a comfortable, biomechanically easily controllable posture when he filled it with water. Holding the filled glass with a thumb-up posture also made it easy to set the glass back down on the table once it was filled with water. The waiter knew that picking up an inverted glass with an uncomfortable thumb-down posture afforded greater comfort and control during the subsequent pouring and placing maneuvers than picking up the glass with a more standard thumb-up posture.

A series of experiments confirmed the generality of this phenomenon and the original interpretation of this so-called end-state comfort effect (Rosenbaum et al. 1990). The experiments were conducted both in the second author's laboratory and in others' laboratories (e.g., Adalbjornsson et al. 2008; Short and Cauraugh 1997). For reviews, see Rosenbaum et al. (2006a) and Rosenbaum (2010).

Whereas the end-state comfort effect was established for unimanual grasps of single objects, other work has shown that people prefer symmetry between the hands for bimanual movements. The term "bimanual symmetry" refers to the tendency to produce similar spatio-temporal movement patterns with the two hands. A wealth of evidence indicates a preference for such symmetry (see Oliveira and Ivry 2008, for a review). For example, when people perform discrete aiming movements for two targets (each target to be reached with each hand), the participants tend to start and complete the aiming movements nearly synchronously (Kelso et al. 1979). The precise coordination between the hands depends to some extent on the individual tasks the two hands must perform and on the visuo-spatial requirements (Riek et al. 2003), but the tendency to start and finish close to synchrony emerges even when the movements to the targets require different distances and/or levels of precision. The tendency for symmetry also emerges when participants are asked to simultaneously produce different spatio-temporal patterns with their two hands (rhythmic bimanual coordination). For example, when participants try to draw a circle with one hand while trying to draw a square with the other hand, the circle tends to become square-like and the square tends to become circle-like, apparently because of an attraction to carry out similar patterns with the two hands at once (Franz et al. 1991).

In the research reviewed above, the preference for endstate comfort was studied in unimanual object-displacement tasks, whereas the preference for bimanual symmetry was studied in two-hand cyclic movement tasks. In the experiments reported here, we studied both constraints at once, in a bimanual task. Joining these two lines of work, let us pit bimanual symmetry against end-state comfort. The questions we asked were whether bimanual symmetry is always more important than end-state comfort or vice versa, and if one constraint is not always more important than the other, then in which circumstances is one constraint more important than the other?

There is precedent for the method we pursued here. Several studies have investigated the importance of bimanual symmetry and end-state comfort when participants manipulated two objects at once, with one object being manipulated with each hand (Fischman et al. 2003; Hughes and Franz 2008; Janssen et al. 2008; Weigelt et al. 2006). In these studies, participants simultaneously grasped two objects (either two cylinders or two CD cases) and transported them to two target locations. The target locations and the required end orientations could either be similar (congruent conditions) or different (incongruent conditions). One question of interest was whether participants used an overhand grip or an underhand grip to grasp the objects. Another question of interest was whether grip type varied as a function of the objects' required end orientations.

These previous studies provided convergent as well as divergent results. A convergent result was that participants adopted object grasps that allowed them to end the object transports comfortably in the congruent conditions. Importantly, planning to end comfortably coexisted with bimanual symmetry in these conditions. A divergent result was obtained in the incongruent conditions, where ending comfortably with both effectors required a break from bimanual symmetry (Hughes and Franz 2008; Janssen et al. 2008; Weigelt et al. 2006). In these cases, the tendency to end comfortably was either preserved (Weigelt et al. 2006), 
reduced in strength (Janssen et al. 2008), or absent (Hughes and Franz 2008).

The inconsistencies in the results just described may indicate that people trade off constraints in a relative rather than in an absolute sense. That is, previous studies were designed in such a way that participants could only plan for one of the two constraints in the incongruent conditions. Possibly, both constraints would show their influence on grasp planning when participants could actually trade them off as they see fit. Such a finding would argue for a changing rather than a strict dominance relation between the endstate comfort constraint and the bimanual symmetry constraint.

We pursued this issue in two experiments reported here. In Experiment 1, we collected data that let us distinguish between the strict dominance relation and the changing dominance relation for end-state comfort and bimanual symmetry using two objects with equal masses and mass distributions. In Experiment 2, we sought to identify the generality of the relation between these constraints that we found in the first experiment, using two objects with unequal masses and mass distributions.

\section{Experiment 1}

In Experiment 1, we asked participants to move two standing plungers from two start locations to two target locations at the same time. The start locations of the plungers were fixed (approximately at waist height), but the heights of the target locations for the two plungers varied (high-high, high-low, low-high, or low-low). The main dependent variable was where participants grasped each plunger. We recorded grasp height (Cohen and Rosenbaum 2004) because it provided a continuous measure of grasp planning. As found by Cohen and Rosenbaum (2004), Rosenbaum et al. (2006b) and Weigelt et al. (2007), grasp heights and target heights are inversely related: Participants grasp plungers low when they plan to place the plungers high, and they grasp plungers high when they plan to place the plungers low. Here, we asked whether this grasp height effect, which has been taken to reflect the end-state comfort effect (see Rosenbaum et al. 2006), would be affected by the congruity of the target heights. If the preference for bimanual symmetry outweighs the preference for end-state comfort, the grasp height effect should be attenuated when the two target heights are different compared to when they are the same. Conversely, if the preference for bimanual symmetry does not outweigh the preference for end-state comfort, the grasp height effect should be unaffected by whether the two target heights are the same or not.
Methods

\section{Participants}

Ten Penn State University students (7 women and 3 men, 19-22 years old) first completed an informed consent form, the short form of the Edinburgh handedness inventory (Oldfield 1971), and a questionnaire about their demographics and neurological status. All participants were right-handed and none reported neurological deficits. All participants were tall enough to easily grasp the plunger shaft at its top when the plunger stood on the top platform. The Penn State University Institutional Review Board approved the experiment and the rights of the participants were protected.

\section{Procedure}

After the participant filled out the forms, s/he was directed to the experimental setup and asked to stand in front of it. The setup, shown in Fig. 1, had three platform heights. The top platform was $120.4 \mathrm{~cm}$ above the floor, the middle platform was $86.4 \mathrm{~cm}$ above the floor, and the bottom platform was $52.4 \mathrm{~cm}$ above the floor. The platforms were shifted relative to one another in depth to minimize the hand path curvature needed to move the plungers without hitting the platforms. The bottom platform was $10 \mathrm{~cm}$ away, the middle platform was $31 \mathrm{~cm}$ away, and the top platform was $52 \mathrm{~cm}$ away from where the participant stood. The start platform had two start locations, and the top and bottom platforms had two target locations. Each start and target
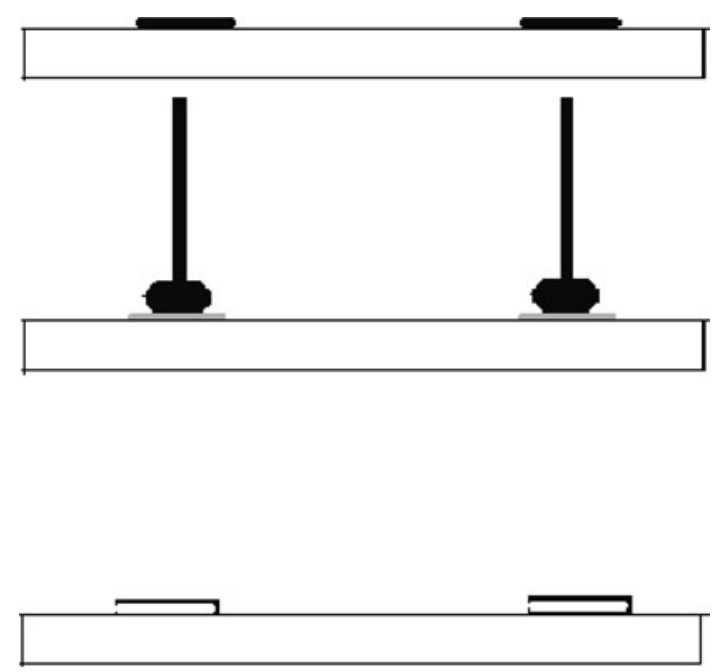

Fig. 1 Overview of the experimental apparatus (not drawn to scale). The apparatus contained three shelves at different heights. The top and bottom shelves each contained one target location for the left and one for the right hand. Two plungers always sat on the middle shelf at the start of a trial 
location was a sheet of paper, 21 by $26 \mathrm{~cm}$. The start locations were gray. The target locations were either black or white. The colors of the two targets on a platform always matched, so the top targets were both black and the bottom targets were both white, or vice versa. The start and target locations were horizontally separated by $5 \mathrm{~cm}$ (edge to edge of their respective paper sheets). The assignment of target colors to target locations stayed constant throughout the experiment for each participant but was randomized over participants.

In each trial, the participant was supposed to grasp the two plungers, the one on the left with the left hand and the one on the right with the right hand, and to move the plungers from their start locations to their respective target locations. We asked participants to lift the plungers from their start locations at the same time and to place them on their target locations at the same time. The start locations were the same in all conditions, namely, on the middle platform. The conditions differed with respect to where the plungers had to be moved. The targets were either both high or both low (the congruent conditions) or were high on the left and low on the right or vice versa (the incongruent conditions). We did not include conditions in which the start locations varied while the target locations stayed constant, as one would predict no grasp height effect in such cases.

Participants started with both of their arms hanging by their sides, standing so their shoulders approximately lined up with the plungers and target pairs. Participants aligned the toes of their feet with a line on the floor $10 \mathrm{~cm}$ away from the lowest platform.

Each plunger had a wooden shaft, $51 \mathrm{~cm}$ long and $23 \mathrm{~mm}$ wide, and a sturdy rubber base, $8 \mathrm{~cm}$ high and $13 \mathrm{~cm}$ wide at its base. The mass of each wooden plunger shaft was $135 \mathrm{~g}$, and the mass of each rubber base was $178 \mathrm{~g}$. The center of mass of each whole plunger was about $1 \mathrm{~cm}$ below the top of the rubber base. Each plunger had a circular disk added to the bottom of its base to allow for recordings of lift-off and placement times from electric switches beneath them (details follow).

On each side of the experimental setup stood a webcam (Logitec Ultra Vision 5000, $30 \mathrm{~Hz}$. frame rate) that focused on the plungers on the start platforms. The left webcam was used to record the grasps of the participant's left hand. The right webcam was used to record the grasps of the participant's right hand. The webcams were placed at approximately the same height as the start platform so the bottom of the rubber base appeared on the bottom of the webcam's field of view and the top of the plunger shaft appeared at the top of the webcam's field of view.

Electric switches at the start and target locations were used to record the times when participants lifted the plungers from the start locations and placed them on the target locations. A digital input/output card (DIO 6356, National
Instruments, Inc.) was used to record the opening and closing of the six switches. A customized MATLAB routine was used to register the switch opening and closing times. These data were stored for later analysis. The recording of pick-up and placement times allowed us to analyze the asynchrony of pick-up times and placement times, as well as movement times for the two hands.

At the beginning of the session, the experimenter told the participant that the experiment focused on how well s/he could time the object displacements to different locations, that is, how well s/he could move the two plungers to their two targets at the same times. The experimenter also told participants to grasp the plungers with a power gripthe kind of grip used to hold a hammer or tennis racquetand to maintain this grip throughout the movement to ensure that the plunger could not slide through their hands during the object transports. The participant was also told that $\mathrm{s} /$ he could move at a comfortable rate.

We wrote a MATLAB program to play a sound file that indicated the colors of the target pair for the particular trial (Rosenbaum 2007). The color of the left target was always announced first, and the color of the right target was always announced second. To ensure that the participant knew the required target locations in a trial, s/he heard the colors of the target pair twice, with a 2-s pause in between. A tone then indicated that the participant could go ahead and move the two plungers to their target locations. No participant appeared to have trouble understanding the instructions.

Each participant completed 6 repetitions of each of the congruent conditions and 16 repetitions of each of the incongruent conditions. In the first block of trials, the participant first completed four trials, two for each of the congruent conditions, followed by 16 trials of one of the incongruent conditions. The second block was identical to the first block, except that participants completed 16 trials of the other incongruent condition. After the second block, the participant completed the last two repetitions for each of the congruent conditions. Thus, each participant completed 44 trials in total. The experiment lasted about $30 \mathrm{~min}$.

\section{Data analysis}

To measure grasp heights, we used the same procedure as Cohen and Rosenbaum (2004), Rosenbaum et al. (2006b), and Weigelt et al. (2007). We used a video playback device to permit frame-by-frame inspection of individual video frames. From the relevant frames, our research assistant clicked on the bottom of the plunger shaft, the top of the plunger shaft, and the highest point of thumb contact on the plunger. These click locations were used to estimate the proportional grasp height, that is, the distance from the bottom of the plunger shaft to the judged highest point of 
thumb contact on the plunger, divided by the total length of the plunger shaft. Inter-rater reliability for this method has been shown in previous work in our lab to be virtually $100 \%$. The proportional grasp height ranged from 0 (grasping directly at the plunger base) to 1 (grasping at the very top of the plunger).

Using the proportional grasp height along the plungers, we calculated the strength of the grasp height effect differently for the congruent and incongruent conditions. For the congruent conditions, we estimated the proportional grasp heights for each hand when both plungers were moved to low target locations (which we expected to be a relatively large value), and we subtracted that value from the proportional grasp heights when both plungers were moved to high target locations (which we expected to be a relatively small value). Thus, the calculation of the grasp height effect for congruent conditions occurred between trials. By contrast, the calculation of the grasp height effect for the incongruent conditions was done within trials. For this measure, the proportional grasp height for the hand that moved a plunger to a high location was subtracted from the proportional grasp height for the hand that moved a plunger to a low target location. For the resulting grasp height differences, values above 0 indicated the presence of a grasp height effect. The reason for using different calculation methods for the congruent and incongruent conditions was that calculating a difference in grasp heights within trials for the congruent conditions was not meaningful because both plungers were moved to the same target heights in these trials.

To calculate the strength of the bimanual symmetry effect in each condition, we calculated the absolute difference in proportional grasp heights between the hands. This calculation was done within a trial, regardless of the target combination that participants performed. For the resulting measure, a value of 0 corresponded to complete bimanual symmetry, whereas positive values indicated deviations from bimanual symmetry; the larger the value, the larger the deviation from symmetry.

For each analysis presented in the next section, we applied a Greenhouse-Geisser correction to the degrees of freedom where appropriate.

Results

\section{Grasp heights}

Figure 2 shows the magnitude of the grasp height effect as a function of repetition number for each of the experimental conditions. The results of the congruent conditions are averaged over the left and right hands. Such averaging was justified because there was no significant difference in the strength of the grasp height effect for the two hands for the

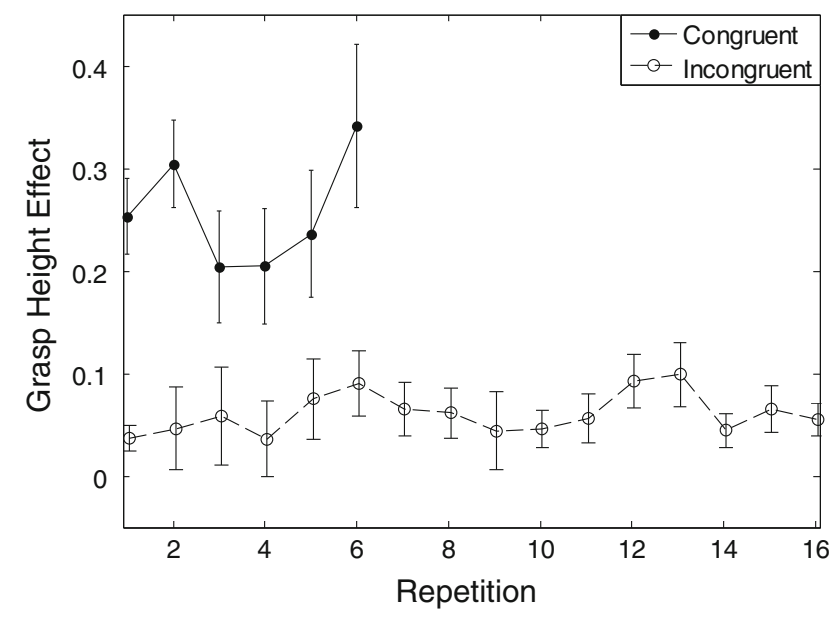

Fig. 2 Mean grasp height effect ( $\pm 1 \mathrm{SE}$ ) for congruent and incongruent conditions of Experiment 1

congruent conditions, $P>.05$. For plotting purposes, we averaged the results for the incongruent conditions. However, the results did indicate a difference between the two incongruent conditions (see analysis below).

To study the strength of the grasp height effect as a function of condition, we conducted a 4 (grasp condition: Congruent Left Hand, Congruent Right Hand, Incongruent High/Low, Incongruent Low/High with the left target indicated first) $\times 6$ (repetition) repeated-measures ANOVA. For the incongruent conditions, we used the first six repetitions. The results indicated a main effect of grasp comparison on the strength of the grasp height effect, $F(3,27)=31.76, P<.01$. Participants showed a stronger grasp height effect in the congruent conditions than in the incongruent conditions, and they showed a stronger grasp height effect for the incongruent high/low condition than for the incongruent low/high condition (.249 for Congruent Left Hand, .265 for Congruent Right Hand, .088 for Incongruent High/Low, and .026 for Incongruent Low/High, respectively). The effect of repetition or the interaction between grasp comparison and repetition did not reach significance, $P>.10$.

\section{Bimanual symmetry}

Figure 3 shows the mean absolute difference in proportional grasp heights for the left and right hands in the congruent and incongruent conditions. Again, we averaged the results of the congruent conditions over the left and right hands, and we averaged the results of the two incongruent conditions as we observed no significant differences between these conditions (see analysis below).

To analyze these data, we performed a 4 (target combination: High/High, Low/Low, High/Low, Low/High with the left target indicated first) $\times 6$ (repetition) repeated-measures 


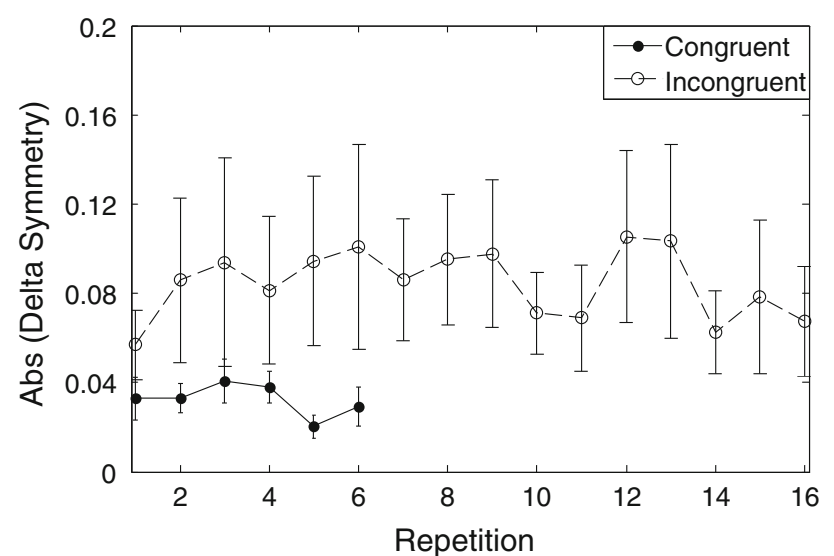

Fig. 3 Mean absolute deviation from bimanual symmetry ( $\pm 1 \mathrm{SE}$ ) for congruent and incongruent conditions of Experiment 1

ANOVA on the mean absolute difference values to test whether the tendency to grasp symmetrically depended on the experimental condition and experience. Again, we used the first six repetitions of the incongruent conditions for this ANOVA. The results indicated a main effect for target combination, $F(1.106,9.952)=4.960, P<.05$. Participants grasped more symmetrically in the congruent conditions than in the incongruent conditions. The results indicated no significant differences between the two congruent conditions or between the two incongruent conditions. Repetition and the interaction between target combination and repetition did not reach significance, $P>.10$.

\section{Timing asynchronies}

To analyze the start and target asynchronies, we calculated the absolute time differences between lifting the two plungers from their start locations (start asynchronies) or placing the plungers on their target locations (target asynchronies). We used the absolute time differences because we were not interested in whether one hand systematically led the other. Instead, the aim of the analyses was to check that participants followed the instruction to move the plungers at approximately the same time. We performed a 4 (target combination; High/High, Low/Low, High/Low, Low/High with the left target indicated first) $\times 6$ (repetition) repeatedmeasures ANOVAs. We used the first six repetitions of the incongruent conditions for these ANOVAs.

Regarding start asynchronies-the absolute differences in the lift times for the left and right plungers from their start positions-the grand mean of those times was only $45 \mathrm{~ms}$. The ANOVA on the absolute differences in the start times revealed a main effect of target combination on the start asynchronies, $F(3,27)=7.379, P<.01$, such that the values were smaller when participants moved both plungers to high locations $(M=31 \mathrm{~ms})$ or when they moved both plungers to low locations $(M=43 \mathrm{~ms})$ than when they moved the left plunger to a high location and the right plunger to a low location $(M=48 \mathrm{~ms})$ or vice versa $(M=56 \mathrm{~ms})$. There were no other significant main effects or interactions for this variable, $P>.10$.

Regarding target asynchronies-the absolute differences in the times for the left and right plungers to reach their target positions-the relevant ANOVA showed that the plungers were brought to the targets with landing asynchronies that depended on target combination, $F(3,27)=$ $11.791, P<.01$. Target asynchronies were smaller when participants moved both plungers to a high location $(M=53 \mathrm{~ms})$ or to a low location $(M=75 \mathrm{~ms})$ than when they moved the left plunger to a high location and the right plunger to a low location $(M=116 \mathrm{~ms})$ or vice versa $(M=175 \mathrm{~ms})$. There were no other significant main effects or interactions for this variable, $P>.10$.

\section{Movement times}

Whereas the two previous sections concerned asynchronies for lifts and placements, respectively, this section concerns the durations of the movements themselves. We studied these times even though they are not independent of the start and target asynchronies because the movement durations were of interest in their own right. We analyzed these times with a 2 (hand; left or right) $\times 4$ (target combination; High/High, Low/Low, High/Low, Low/High) $\times 6$ (repetition) repeated-measures ANOVA. The ANOVA yielded a significant main effect for hand, $F(1,9)=29.800, P<.01$, such that left-hand object transports $(M=1,340 \mathrm{~ms})$ took longer than right-hand object transports $(M=1,256 \mathrm{~ms})$. The main effect for target combination also reached significance, $F(1.994,17.945)=6.943, P<.01$, such that object transports took less time when both objects were moved to a high location $(M=1,122 \mathrm{~ms})$ or to a low location ( $M=1,265 \mathrm{~ms})$ than when the left plunger was moved to a high location while the right plunger was moved to a low location $(M=1,390 \mathrm{~ms})$ or vice versa $(M=1,415 \mathrm{~ms})$. There were no other significant main effects or interactions for this variable, $P>.10$.

\section{Discussion}

In the first experiment, we sought to determine whether two constraints for grasp planning in object manipulation have a strict dominance relation or a changing dominance relation. The two constraints we studied were a preference for endstate comfort on the one hand and a preference for bimanual symmetry on the other. Previous studies have provided conflicting results about the relative importance of these two constraints. We hypothesized that these inconsistencies did not arise from limitations or inconsistencies in the 
previous studies, but instead arose because the constraints have a changing rather than a strict constraint dominance relation.

To test this hypothesis, we focused on the grasp heights that participants used to move two plungers to the same or different heights. The main hypotheses we tested were as follows: If the preference for bimanual symmetry outweighs the preference for end-state comfort, the grasp height effect should be attenuated when the two target heights differ. Conversely, if the preference for bimanual symmetry does not outweigh the preference for end-state comfort, the grasp height effect should be unaffected by whether the two target heights are the same.

In the congruent conditions, where the heights of the two plungers' targets were the same, there was a strong grasp height effect and a strong symmetry effect. Participants grasped the plungers approximately symmetrically and closer to their tops when they moved the plunger to low target locations, whereas they grasped the plungers approximately symmetrically and closer to their bottoms when they moved the plungers to the high target locations. Thus, the adopted grasps followed end-state comfort planning and bimanual symmetry in the congruent conditions. This outcome makes the interpretation of the grasp height effect in the incongruent conditions meaningful.

In the incongruent conditions, the grasp height effect was present but was much smaller than in the congruent conditions. Participants also deviated significantly from grasping symmetrically in these conditions. Thus, participants exhibited a trade-off between end-state comfort and bimanual symmetry. Neither end-state comfort nor bimanual symmetry completely dominated the other. This outcome is consistent with a changing dominance relation rather than a strict dominance relation for end-state comfort and bimanual symmetry in bimanual grasp planning.

\section{Experiment 2}

Experiment 2 was designed to address a concern about the first experiment and was also designed to test the generality of the conclusion from the first experiment. We were concerned that the outcome of the first experiment may have depended on the need to look back and forth between the spatially disparate target locations more in the incongruent conditions (high-low or low-high targets) than in the congruent conditions (high-high or low-low targets). Sequential looking behavior has been shown to yield asynchronous timing in bimanual reach-to-grasp tasks (Bingham et al. 2008), lending credence to this possibility. We addressed this concern in the second experiment by asking participants to grasp the objects either simultaneously, as they did in Experiment 1, or sequentially before moving the two objects at the same time to their respective target sites.

The other change to the method was to have participants move plungers of different weights; in Experiment 1 participants moved plungers of equal weight. Previous research has shown that people are sensitive to center of mass in object grasps (Lederman and Wing 2003; Lukos et al. 2007; Serrien et al. 2001a, b). We reasoned that if the prioritization of end-state comfort and bimanual symmetry is context-dependent, the relative importance of these factors might change when the weights of the objects were different (Experiment 2) when compared to when they were the same (Experiment 1).

\section{Methods}

\section{Participants}

Sixteen Penn State students (9 women and 7 men, age 1824 years) participated. They first completed the same forms as in Experiment 1. None reported any neurological deficits and all were right-handed. All participants were tall enough to comfortably reach the top of the plunger shaft when it was on the top platform. The Penn State University Institutional Review Board approved the experiment, and the rights of all participants were protected.

\section{Procedure}

The experimental method for Experiment 2 was the same as for Experiment 1, except that one of the plungers was heavier than the other in all trials. To achieve this difference in weight, we added a cylindrical extension to the top of each plunger shaft. This extension was a PVC pipe that was $23 \mathrm{~cm}$ long and $3.3 \mathrm{~cm}$ in diameter. The $\mathrm{PVC}$ pipe was connected to the top of the plunger shaft with silver electrical tape, and the PVC pipe was wrapped with electrical tape to make it look more homogeneous. Altogether, the plunger shaft with the added PVC pipe was $70 \mathrm{~cm}$ long. The PVC pipe weighed 128 gram. For each participant, an additional 400 gram of American nickel coins was added to one of the PVC pipes, thus creating a setting in which one plunger was heavier than the other. The center of mass of the plunger without a weight was located at approximately $30.0 \%$ of the plunger shaft. Adding the weight moved the center of mass up along the plunger to approximately $74.4 \%$ of the plunger shaft. The heavier plunger always stood on one side for all trials for a given participant. The side of the heavier plunger was counterbalanced between participants.

Before the start of the experiment, participants were told to grasp and lift the two plungers once to ensure that they were aware of the plungers' respective weights. Participants 
then completed two blocks of 24 trials each. The order of the blocks was counterbalanced over participants. In one block, participants were instructed to grasp the plungers one at a time (sequential grasping), after which they were to move the plungers at the same time. In the other block, participants grasped the plungers at the same time (simultaneous grasping), and also to move the plungers at the same time. We did not include conditions in which participants moved the plungers sequentially because our focus was on the nature of the planned grasps for the same motor act (simultaneous motions of the two plungers to their respective targets) depending on the challenges to the planning system prior to this motor act.

The method of presentation of the target sequence for trials in the simultaneous grasping block was identical to the method used in Experiment 1. For the sequential grasping trials, participants first heard the color name for the left target followed by the color name for the right target. After a 2-s pause, participants again heard the color name of the left target, whereupon they were supposed to grasp the left plunger. After another 2-s pause, participants heard the color for the right target again, whereupon they were supposed to grasp the right plunger. One second later, a tone sounded, indicating that the participant could move both plungers simultaneously to their respective target locations.

Participants completed each congruent condition during trials 1 and 2 of any given block of trials, and again during the 13th and 14th trials in any given block. In trials 3-12 of a block, participants performed repetitions of one incongruent condition (i.e., Up/Down or Down/Up), and in trials 15-24 of a block they completed repetitions of the other incongruent condition. The order of the congruent and incongruent conditions within a block was randomized across participants.

\section{Results}

\section{Grasp heights}

Figure 4 shows the magnitude of the grasp height effect $( \pm 1 \mathrm{SE})$ as a function of repetition number for each of the experimental conditions. We averaged the results of the congruent conditions for the left and right hand, and we averaged the results for the incongruent conditions for cases in which participants moved the object on the left up and the object on the right down, and vice versa. Such averaging was justified because the results indicated no significant differences between these conditions (see analysis below). The calculation of the strength of the grasp height effect in different conditions was the same as that used in Experiment 1.

To analyze the strength of the grasp height effect as a function of condition and repetition, we performed a 2 (grasp condition; sequential or simultaneous) $\times 4$ (grasp comparison; Congruent Left Hand, Congruent Right Hand, Incongruent High/Low, Incongruent Low/High with the left target indicated first) $\times 6$ (repetition) repeated-measures ANOVA. The results indicated a main effect of grasp combination, $F(1.653,24.800)=4.998, P<.01$, such that participants showed a stronger grasp height effect for the congruent conditions $(M=.256$ and $M=.272$ for the left and right hand, respectively) than for the incongruent conditions $(M=.182$ and $M=.159$ for the Incongruent High/Low and Incongruent Low/High, respectively). The results also indicated a main effect for grasp condition, $F(1,15)=5.014, P<.05$, such that participants showed a stronger grasp height effect when they grasped both plungers simultaneously $(M=.256)$ than when they grasped both plungers sequentially $(M=.179)$. Finally, there was a main effect for repetition, $F(2.258,33.867)=7.492, P<.01$, such
Fig. 4 Mean grasp height effect ( $\pm 1 \mathrm{SE}$ ) for congruent and incongruent conditions of Experiment 2 when grasps were to be made sequentially (left panel) or simultaneously (right panel)

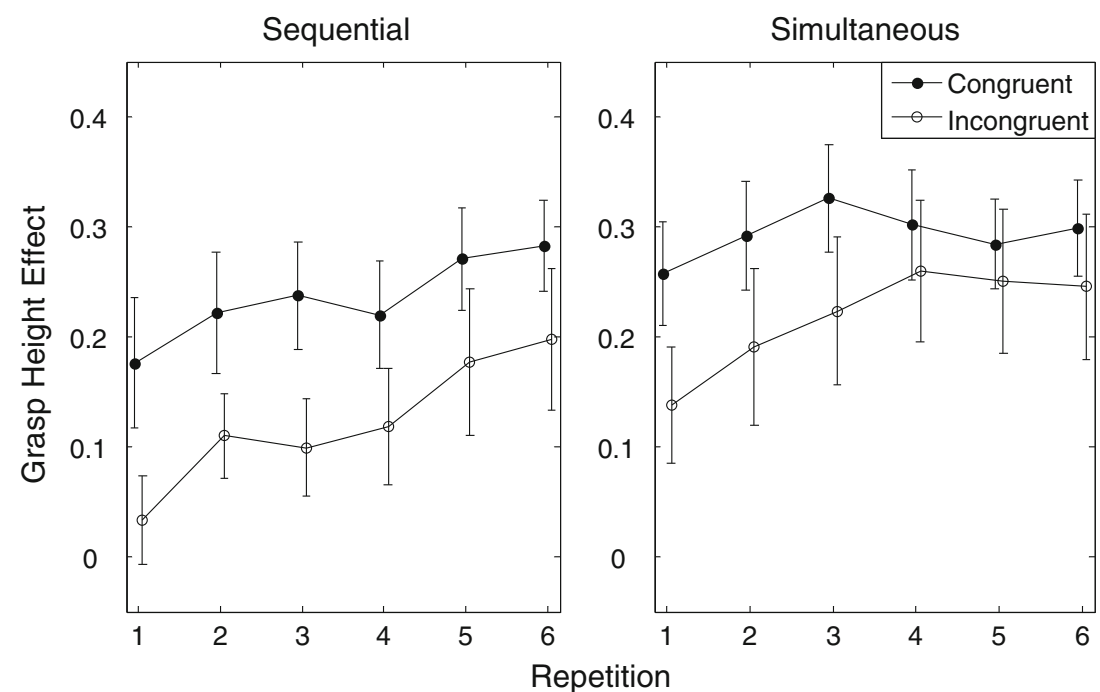


that the grasp height effect grew larger over repetitions. None of the interactions approached significance.

To test whether participants increasingly relied on endstate comfort planning over repetitions when doing so implied compromising bimanual symmetry, we performed an additional ANOVA on the repetitions for just the incongruent conditions. If participants assigned more weight to plan for end-state comfort with increased experience, one would predict a significant effect of repetition in the incongruent condition. A 2 (grasp condition; sequential or simultaneous) $\times 2$ (grasp comparison; Incongruent High/ Low, Incongruent Low/High with the left target indicated first) $\times 6$ (repetition) repeated-measures ANOVA confirmed this prediction and showed no other significant main effects or interactions. Thus, participants showed a substantial increase in the strength of the grasp height effect over repetitions for the incongruent conditions, $F(1.679$, 25.182) $=5.795, P<.01$.

\section{Bimanual symmetry}

Figure 5 shows the mean absolute difference in proportional grasp heights for the left and right hand in the congruent and incongruent conditions. For this measure, larger positive values correspond to larger deviations from grasping symmetrically. To analyze how close to symmetry participants grasped the plungers in different conditions, we performed a 2 (grasp condition; sequential or simultaneous) $\times 4$ (grasp comparison; Congruent Left Hand, Congruent Right Hand, Incongruent High/Low, Incongruent Low/High with the left target indicated first) $\times 6$ (repetition) repeated-measures ANOVA. The results indicated main effects for grasp comparison,
$F(1.590,23.850)=3.230, P<.05$, and repetition, $F(1.841$, $27.619)=5.972, P<.01$, an interaction between grasp comparison and repetition, $F(4.078,61.166)=2.614, P<.05$, and an interaction between grasp condition and grasp comparison, $F(1.834,27.511)=3.103, P<.05$. Whereas the symmetry effect stayed approximately constant for the congruent conditions, participants showed a large increase in their departure from symmetry over repetitions for the incongruent conditions. In addition, the departure from symmetry for the incongruent conditions was stronger when participants grasped the plungers simultaneously $(M=.218)$ than when participants grasped the plungers sequentially $(M=.123)$. The results indicated no other significant main effects or interactions, $P>.05$.

\section{Timing asynchronies}

To analyze the absolute start and target asynchronies, we performed 2 (grasp timing; sequential or simultaneous) $\times 4$ (target combination; High/High, Low/Low, High/Low, Low/High with the left target indicated first) $\times 6$ (repetition) repeated-measures ANOVAs.

Regarding start asynchronies, the ANOVA indicated a significant main effect of target combination on the absolute start asynchronies, $F(3,39)=9.933, P<.01$, such that participants started their movements closest to synchrony when moving both plungers to a low location $(M=30 \mathrm{~ms})$ compared to moving both plungers to a high location ( $M=32 \mathrm{~ms}$ ), moving the left plunger to a high location and the right plunger to a low location $(M=37 \mathrm{~ms})$, and moving the left plunger to a low location and the right plunger to a high location $(M=48 \mathrm{~ms})$. Start asynchronies stayed relatively constant in most conditions, but decreased over
Fig. 5 Mean absolute deviation from bimanual symmetry $( \pm 1$ $\mathrm{SE})$ for congruent and incongruent conditions of Experiment 2 when grasps were to be made sequentially (left panel) or simultaneously (right panel)
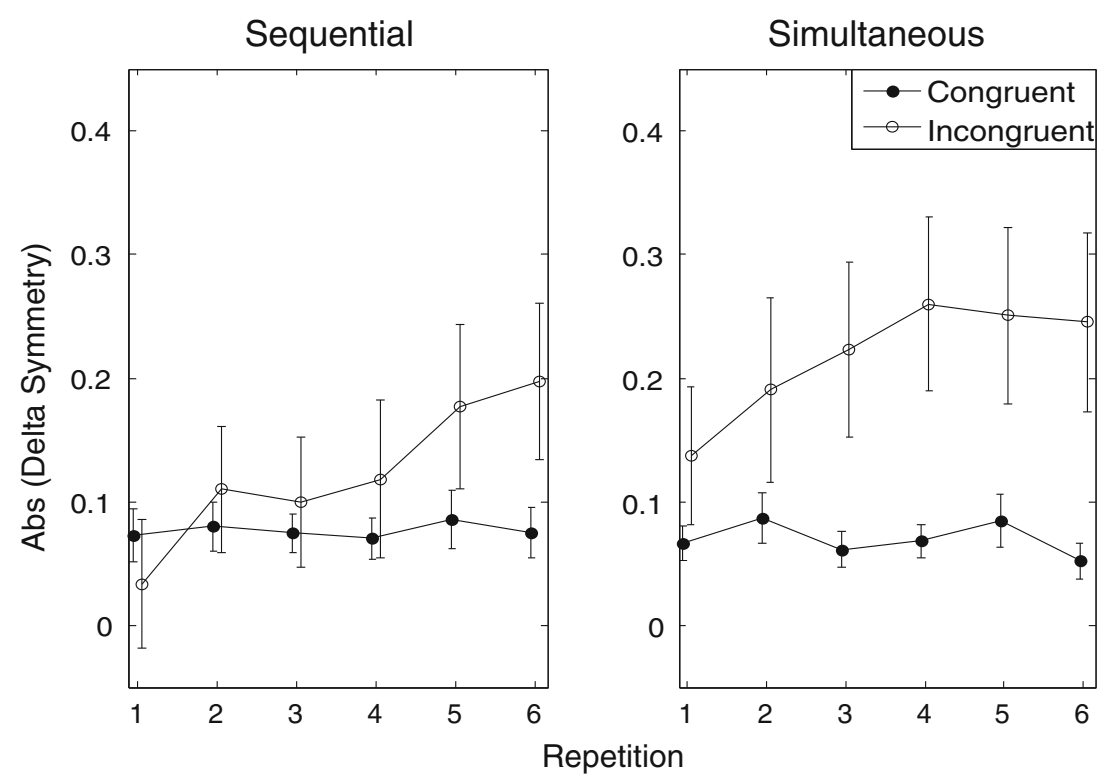
repetitions when moving the left plunger down and the right plunger up, $F(15,195)=2.094, P<.05$. The results showed no other significant main effects or interactions, $P>.10$.

Regarding target asynchronies, the ANOVA indicated a significant main effect of target combination on the absolute target asynchronies, $F(1.934,29.014)=15.865, P<.01$, such that participants completed their movements closest to synchrony when moving both plungers to a high location $(M=71 \mathrm{~ms})$ compared to moving both to a low location $(M=101 \mathrm{~ms})$, moving the left plunger to a high location and the right plunger to a low location ( $M=177 \mathrm{~ms}$ ), and moving the left plunger to a low location and the right plunger to a high location $(M=187 \mathrm{~ms})$. Participants' timing for the congruent conditions was significantly better than for the incongruent conditions. The differences between the two conditions in each of these cases did not reach significance. Target asynchronies decreased over repetitions, $F(3.123,46.844)=4.100, P<.05$. The results revealed no other significant main effects or interactions, $P>.10$.

\section{Movement times}

A 2 (grasp timing; sequential or simultaneous) $\times 2$ (hand; left or right) $\times 4$ (target combination; High/High, Low/ Low, High/Low, Low/High with the left target indicated first) $\times 6$ (repetition) repeated-measures ANOVA indicated a significant main effect for hand on movement times, $F(1,8)=10.045, P<.05$, such that object transports for the left hand $(M=1,420 \mathrm{~ms})$ took longer than object transports for the right hand $(M=1,308 \mathrm{~ms})$. The main effect for target combination on movement times also reached significance, $F(3,24)=15.056, P<.01$, such that object transports took shortest when moving both objects to a high location ( $M=1,237 \mathrm{~ms})$, followed by moving both objects to a low location $(M=1,354 \mathrm{~ms})$, moving the left plunger to a low location while moving the right plunger to a high location $(M=1,361 \mathrm{~ms})$, and moving the left plunger to a high location while moving the right plunger to a low location $(M=1,504 \mathrm{~ms})$. Movement times decreased with more repetitions, $F(2.016,16.127)=16.127, P<.01$. Movement times for the left hand decreased more strongly than for the right hand with increasing numbers of repetitions, $F(2.069,16.549)=4.190, P<.05$. The results did not reveal any other significant main effects or interactions, $P>.10$.

\section{Cross-experiment analysis}

To further examine the extent to which the preference for end-state comfort and bimanual symmetry during grasp planning was context-dependent, we performed a crossexperiment analysis to compare the results of Experiments
1 and 2. In particular, we conducted two 4 (Condition) $\times 6$ (Repetition) ANOVAs, one on the grasp height effect and one on the bimanual symmetry effect, in which we included Experiment as a between-subject factor. We used the first 6 repetitions of the congruent conditions of Experiment 1, and all 6 repetitions of the congruent conditions of Experiment 2 and the incongruent conditions of Experiments 1 and 2. We discuss the presence of main effects and interactions for the factor Experiment below as this factor relates to effects of task context that go beyond the analyses already reported for Experiments 1 and 2.

The results of the ANOVA on the grasp height effect failed to support an interaction (assuming alpha =.05) between Experiment and Condition, $F(1.640,39.351)=$ 2.593, $P=.097)$, despite the tendency for the grasp height effect to be somewhat larger in Experiment 2 than in Experiment 1 for the incongruent conditions but not for the congruent conditions. Similarly, there was a nonsignificant interaction between Repetition and Experiment, $F(2.755,66.114)=2.549, P=.068)$, though the grasp height effect increased for the unequally weighted plungers (E2) but not for the standard plungers (E1).

A similar ANOVA on the bimanual symmetry effect showed a main effect for Experiment, such that participants showed a larger departure from symmetry when they moved two plungers of unequal weight compared to two plungers of equal weight $(\mathrm{E} 2), F(1,24)=5.321, P<.05$. Experiment did not interact significantly with the other factors in this analysis.

\section{Discussion}

In Experiment 2, we asked whether the changing constraint relationship found for end-state comfort and bimanual symmetry in Experiment 1 held across changes in task context, where those changes were, at least in terms of experimental design, biomechanical or behavioral. The biomechanical change in task context was the use of plungers with different mass distributions in Experiment 2 as compared to Experiment 1 . The behavioral change in task context was the requirement to grasp the plungers either sequentially or simultaneously before moving the plungers, simultaneously in both cases, to their respective targets.

As in Experiment 1, neither the end-state comfort constraint nor the bimanual symmetry constraint strictly dominated grasp planning in any condition. Consistent with the hypothesis that the relative importance of the two constraints could change, we found that end-state comfort became more important in the incongruent conditions than in the congruent conditions, as evidenced by the increase in the grasp height effect and decrease in the symmetry effect over repetitions in these conditions. Regarding the effects of grasping the plungers sequentially or simultaneously, we 
obtained the same general pattern of results in both conditions. The only difference was that participants showed a somewhat stronger grasp height effect and a somewhat weaker symmetry effect in the incongruent conditions when they grasped both plungers at the same time than when they grasped the plungers one at a time. Nonetheless, the fact that the sequential-grasp and simultaneous-grasp conditions in Experiment 2 yielded similar results suggests that not being able to look at one plunger at a time during object manipulation (see Bingham et al. 2008; Riek et al. 2003) is the not the sole basis for the changing dominance relation for grasp planning observed in Experiment 1.

The results of our cross-experiment analysis provide further support for the claim that bimanual grasp planning relies on a changing dominance relation, as participants changed the emphasis they placed on end-state comfort versus bimanual symmetry depending on whether they transported objects of similar or different weight.

\section{General discussion}

In this study, we sought to determine whether two constraints for object manipulation follow a strict dominance relation or a changing dominance relation. The two constraints we studied were end-state comfort and bimanual symmetry. We found that neither end-state comfort nor bimanual symmetry consistently dominated the other. Our results clearly favor a changing dominance view rather than a strict dominance view of the relation between end-state comfort and bimanual symmetry.

In the remainder of the General Discussion, we consider the implications of our results for the understanding of motor planning vis à vis biomechanical demands, visuospatial demands, and the understanding of perceptual-motor skill learning.

With respect to biomechanical demands, we found that the relative emphasis on end-state comfort and symmetry was different in Experiment 2, where the plungers had different mass distributions, than in Experiment 1, where the plungers had the same mass distributions. Participants in Experiment 1 were more inclined to grasp the plungers in similar locations than were participants in Experiment 2. Because the mass distributions of the two plungers were the same in Experiment 1, grasping the plungers in similar locations let the hands experience similar dynamics when the plungers were transported to their targets, or at least this was very plausibly the case in the congruent (same-targetheight) conditions. Sensitivity to the dynamics of object manipulation has been shown for unimanual grasp selection (Lederman and Wing 2003; Lukos et al. 2007), and a tendency for the two hands to show similar dynamics has been shown for grip forces used to lift objects of different weights (Serrien and Wiesendanger 2001a, b).

With respect to visuo-spatial demands, we sought to understand the interplay between such demands and object manipulation, respecting the fact that planning for action could be affected by visuo-spatial demands and may also be done in the service of directing visuo-spatial attention. For example, when one turns to look at a target of interest, one plans and controls the associated movements to heighten the attention that can be paid to the target and to increase the extent to which the target can be seen. This in turn may influence the planning of object grasps. Our results indicate that instructing participants to grasp the plungers sequentially or simultaneously had only a small effect on the relative strength of end-state comfort and bimanual symmetry. Thus, our results suggest that participants established the relative importance of bimanual symmetry and end-state comfort constraints for grasp planning before they reached for the plungers, as visuo-spatial demands would likely have influenced the results otherwise (Bingham et al. 2008; Riek et al. 2003).

Finally, our results contribute in a useful way, we feel, to the understanding of perceptual-motor skill learning. As one gains skill, one may become increasingly sensitive to nuances in performance, including the perceptual consequences of performance. But how does that increasing sensitivity come about? We speculate that it comes about by changing the relative importance of different action constraints. Initially, all the possible constraints are assigned equal weight, or the possible constraints are assigned weights that comprise a reasonable guess about their relative importance based on the similarity of the task to be performed to other, more familiar, tasks. Over time, the relative importance of the constraints may change in a way that promotes more effective performance. According to this account, developing a perceptual-motor skill is as much about defining and redefining the task to be performed as it is about performing the task. When a performer learns to perform a task better than $s / h e$ did before, it may be because s/he changed what $\mathrm{s} / \mathrm{he}$ was actually trying to do. This is a different view of skill learning than ones that are currently in the literature (cf. Rosenbaum 2010). The present study suggests that changing priorities in task performance is a rapid and flexible process, as is probably needed for adaptive learning. The latter comments are speculative, but the results reported here or others obtained in the same general way-pitting alternative constraints against each other and seeing how their relative importance changes with practice-may allow the speculation to be tested in a more rigorous fashion as research continues in this domain. 
Acknowledgments The authors thank Geoffrey Bingham, Richard Carlson, Mark Fischman, Frank Hillary, Ruud Meulenbroek, Marc Mon-Williams, and Dagmar Sternad for helpful comments, and we thank Louis Costanzo, Cassandra Johnson, Mike Roche, and Kate Wagner for help with data collection. The research was supported by grants from the Penn State Social Science Research Institute, the Penn State College of Liberal Arts Office of Research and Graduate Studies, the National Science Foundation, and the National Institutes of Health.

Open Access This article is distributed under the terms of the Creative Commons Attribution Noncommercial License which permits any noncommercial use, distribution, and reproduction in any medium, provided the original author(s) and source are credited.

\section{References}

Adalbjornsson CF, Fischman MG, Rudisill ME (2008) The end-state comfort effect in young children. Res Q Exerc Sport 79:36-41

Bingham GP, Hughes K, Mon-Williams M (2008) The coordination patterns observed when two hands reach-to-grasp separate objects. Exp Brain Res 184(3):283-293

Cohen RG, Rosenbaum DA (2004) Where objects are grasped reveals how grasps are planned: generation and recall of grasps. Exp Brain Res 157:486-495

Fischman MG, Stodden DF, Lehman DM (2003) The end-state comfort effect in bimanual grip selection. Res Q Exerc Sport 74:17-24

Franz EA, Zelaznik HN, McCabe G (1991) Spatial topological constraints in a bimanual task. Acta Psychol 77:137-151

Hughes CML, Franz EA (2008) Goal-related planning constraints in bimanual grasping and placing of objects. Exp Brain Res 188:541-550

Janssen L, Beuting M, Meulenbroek R, Steenbergen B (2008) Combined effects of planning and execution constraints on bimanual task performance. Exp Brain Res 192:61-73

Kelso JAS, Southard DL, Goodman D (1979) On the co-ordination of two-handed movements. J Exp Psychol Hum Percept Perform 5:229-238

Lederman SJ, Wing AM (2003) Perceptual judgment, grasp selection, and object symmetry. Exp Brain Res 152:156-165

Lukos J, Ansuini C, Santello M (2007) Choice of contact points during multidigit grasping: effect of predictability of object center of mass location. J Neurosci 27:3894-3903
Oldfield RC (1971) The assessment and analysis of handedness: the Edinburgh inventory. Neuropsychologia 9:97-113

Oliveira FTP, Ivry RB (2008) The representation of action: insights from bimanual coordination. Curr Dir Psychol Sci 17(2):130-135

Prince A, Smolensky P (1997) Optimality: from neural networks to universal grammar. Science 275:1604-1610

Riek S, Tresilian JR, Mon-Williams M, Coppard V, Carson RC (2003) Bimanual aiming and overt attention: one law for two hands. Exp Brain Res 153:59-72

Rosenbaum DA (2007) MATLAB for behavioral scientists. Lawrence Erlbaum Associates, Mahwah

Rosenbaum DA (2010) Human motor control, 2nd edn. Academic Press/Elsevier, San Diego

Rosenbaum DA, Marchak F, Barnes HJ, Vaughan J, Slotta J, Jorgensen MJ (1990) Constraints for action selection: overhand versus underhand grips. In: Jeannerod M (ed) Attention and performance XIII-motor representation and control. Erlbaum, Hillsdale, pp 321-342

Rosenbaum DA, Loukopoulos LD, Meulenbroek RGJ, Vaughan J, Engelbrecht SE (1995) Planning reaches by evaluating stored postures. Psychol Rev 102:28-67

Rosenbaum DA, Meulenbroek RGJ, Vaughan J, Jansen C (2001) Posture-based motion planning: applications to grasping. Psychol Rev 108:709-734

Rosenbaum DA, Cohen RG, Meulenbroek RG, Vaughan J (2006a) Plans for grasping objects. In: Latash M, Lestienne F (eds) Motor control and learning over the lifespan. Springer, New York, pp 9-25

Rosenbaum DA, Halloran E, Cohen RG (2006b) Grasping movement plans. Psychon Bull Rev 13:918-922

Serrien DJ, Wiesendanger M (2001a) A higher-order mechanism overrules the automatic grip-load force constraint during bimanual asymmetric movement. Behav Brain Res 118:153-160

Serrien DJ, Wiesendanger M (2001b) Bimanual organization of manipulative forces: evidence from erroneous feedforward programming of precision grip. Eur J Neurosci 13:1825-1832

Short MW, Cauraugh JH (1997) Planning macroscopic aspects of manual control: end-state comfort and point-of-change effects. Acta Psychol 96:133-147

Weigelt M, Kunde W, Prinz W (2006) End-state comfort in bimanual object manipulation. Exp Psychol 53:143-148

Weigelt M, Cohen RG, Rosenbaum DA (2007) Returning home: locations rather than movements are recalled in human object manipulation. Exp Brain Res 149:191-198 